\title{
Spectrophotometric Determination of Sertaconazole Nitrate in Pharmaceutical Dosage Form using Quality by Design (QbD) Framework
}

Sagar Suman Panda ${ }^{1^{*}}$, Sarwar Beg ${ }^{2^{*}}$, Ravi kumar Venkata Varaha Bera ${ }^{1}$ and Aruneema Gain ${ }^{1}$

${ }^{1}$ Department of Pharmaceutical Analysis and Quality Assurance, Roland Institute of Pharmaceutical Sciences, Berhampur, Odisha, India

${ }^{2}$ Product Development Research, Jubilant Generics Ltd., Noida, Uttar Pradesh, India

\begin{abstract}
An accurate and reliable ultra-violet spectrophotometric method was developed based on the Quality by Design framework, for the determination of sertaconazole nitrate in solid oral dosage form. Sampling interval and scanning speed were the two critical method variables, which were also evaluated by design of experiment approach, for establishing method robustness using optimization technique. Sertaconazole shows absorption maximums at 260 $\mathrm{nm}, 293 \mathrm{~nm}$ and $302 \mathrm{~nm}$, respectively using methanol as solvent. Good linearity was obtained for sertaconazole over concentration of $10-60 \mu \mathrm{g} / \mathrm{ml}$ with $R^{2}>0.99$ at all the three wavelengths. The results of validation study were within acceptance limits as per $\mathrm{ICH}$ guidelines. The $\mathrm{QbD}$ instigated method for determination of sertaconazole nitrate can be highly effective for routine analysis purpose.
\end{abstract}

Keywords: Sertaconazole; UV spectrophotometry; Quality by design; Central composite design; Robustness

\section{Introduction}

Sertaconazole nitrate (SRT), $( \pm)-1\{2, \quad$ 4-dihydro- $\beta$ - $[(7-$ chlorobenzo[b]thien-3-yl)-methoxy] phenethyl\} imidazole nitrate (Figure 1) is an antifungal drug used in treating candidiasis, seborrhoeic dermatitis and pytiriasis versicolor $[1,2]$.

Sertaconazole nitrate in dosage forms is estimated by a few UVvisible spectroscopic methods [3-5]. The methods reported in literature possessed drawbacks like lack of information on sensitivity studies, molar extinction coefficients, and not addressing the additional peaks obtained in the spectrum, etc. Moreover; these reported methods fail to establish the robustness of the method. Thus, efforts were made to develop a Quality-by-Design (QbD) driven superior ultra-violet spectrophotometric method to determine sertaconazole nitrate in tablets.

By employing a sound science-based risk assessment, QbD framework provides a clear knowledge on method variables, which may affect the analytical attributes [6]. Figure 2 depicts the various steps involved in analytical $\mathrm{QbD}$ approach for development of robust methods.

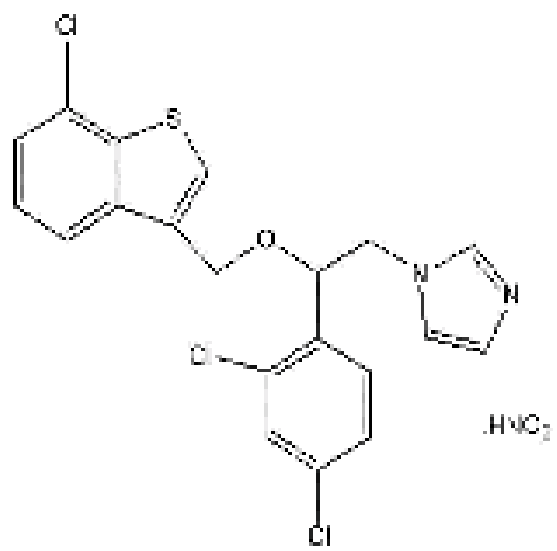

Figure 1: Chemical structure of sertaconazole nitrate.

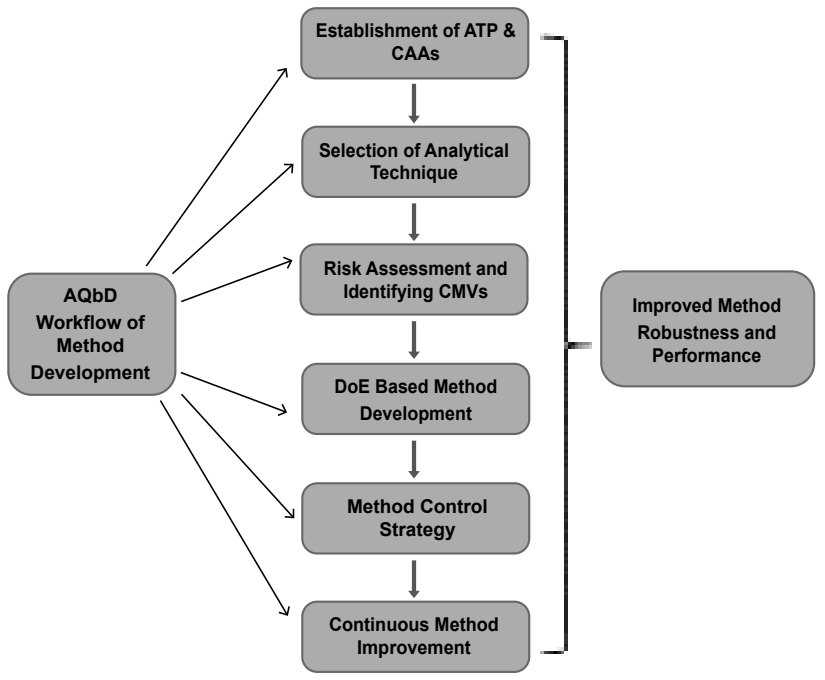

Figure 2: The methodical analytical $\mathrm{QbD}$ approach for method development.

In the present study, the analytical target profile (ATP) and critical analytical attribute (CAA) for developing a robust UV spectrophotometric method were defined rationally. Suitable risk

*Corresponding authors: Sagar Suman Panda, Asst. Professor, Department of Pharmaceutical Analysis and Quality Assurance, Roland Institute of Pharmaceutical Sciences, Khodasingi, Berhampur-760010, Odisha, India, Tel: +91-9438040646; E-mail: sagarguddu2002@gmail.com

Sarwar Beg, Research Scientist, Product Development Research, Jubilan Generics Ltd., Noida-201301, Uttar Pradesh, India, Tel: +91-9437770090; E-mail: sarwar.beg@gmail.com

Received August 23, 2017; Accepted September 22, 2017; Published September 29, 2017

Citation: Panda SS, Beg S, Ravi Kumar VVB, Gain A (2017) Spectrophotometric Determination of Sertaconazole Nitrate in Pharmaceutical Dosage Form using Quality by Design (QbD) Framework. J Bioanal Biomed 9: 235-239. doi:10.4172/1948-593X.1000185

Copyright: @ 2017 Panda SS, et al. This is an open-access article distributed under the terms of the Creative Commons Attribution License, which permits unrestricted use, distribution, and reproduction in any medium, provided the original author and source are credited. 
assessment followed by risk management using experimental design based investigation of critical method variables (CMVs) for their effect on CAA was carried out. Further, method control strategies were framed for achieving continuous improvement of method performance. Therefore, based on the above intent successful attempts were made to accentuate the QbD-based development and validation of a robust spectrophotometric method for rapid and economic determination of sertaconazole in pharmaceutical formulations [7].

\section{Materials and Methods}

\section{Reagents and chemicals}

Gift sample of standard sertaconazole nitrate drug (purity $>99.85 \%$ ) was received from Glenmark Pharmaceuticals Ltd., India. Methanol (Merck Ltd., Mumbai, India) was used as solvent. Marketed tablet formulation of sertaconazole $(500 \mathrm{mg}$ ) was assessed for its purity determination.

\section{Instrumentation}

A SHIMADZU 1800 UV spectrophotometer (Kyoto, Japan) along with a high-precision analytical balance and an ultrasonicatior (Enertech, India) was used for the purpose.

\section{Establishment of ATP}

Investigation of literature and analyte profile helped towards the establishment of method objective. This primarily constituted developing a robust and cost-effective analysis of sertaconazole in tablet formulation. In order to meet the ATP, an UV spectrophotometric method was selected considering absorbance of sertaconazole as CAA.

\section{Risk analysis and it's management}

To understand the causal-effect relation between the potential method variables and CAA, a typical fish-bone diagram can be used. Hence, a fish- bone diagram addressing various method variables affecting CAA was prepared (Figure 3). A Control-NoiseExperimental(C-N-X) approach along with a suitable assessment matrix was employed for discovering the highest risk variables controlling the CAA. Scores were assigned to each variable and total score were evaluated to identify the CMVs. Variables viz. types of solvent, detection, scanning speed, sampling interval, sample intactness, etc. was evaluated for this purpose. Design of experiment approach was utilized for investigation and subsequent optimization of the sorted CMVs.

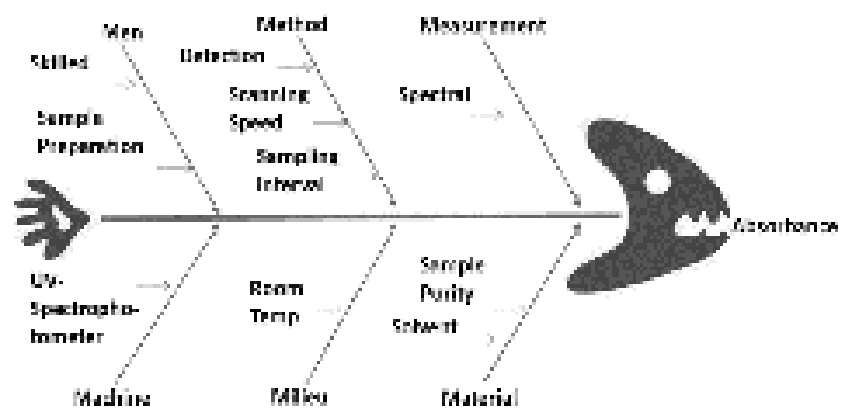

Figure 3: Typical fish-bone diagram depicting cause-effect relationship between method variables and CAA.

\section{Optimization of robustness study and data analysis}

Central Composite Design (CCD) was applied for identifying optimum method conditions and to ensure robustness. A robust design space was established after selecting a CCD domain, with the intent of obtaining fewer experiments (ten experiments with two center points) and greater. Absorbance of standard sertaconazole $20 \mu \mathrm{g} / \mathrm{ml}$ at 260 $\mathrm{nm}, 293 \mathrm{~nm}$ and $302 \mathrm{~nm}$ was measured as the response variable and assessed as per CCD.

Fitting the experimental data to a suitable mathematical model followed by exhaustive data analysis was carried out by using JMP software. Polynomial equations in the form of prediction expression were prepared considering the coefficients of significant model terms with probability value less than 0.05 according to Analysis of Variance (ANOVA). Model suitability was evaluated through lack of fit and correlation coefficient values. Two-dimensional contour (2-D) and three-dimensional response surface (3-D) profiler were analyzed to establish the relationship between CMVs and CAA. The optimum method conditions were optimized by numerical and graphical mode within the robust design space.

\section{Control strategy}

Control strategies were defined based on the results obtained for CAA. Control strategy was instituted for all the CMVs as per results obtained from numerical and graphical optimization.

\section{Preparation of stock and working solution}

Standard stock solution of concentration $1000 \mu \mathrm{g} / \mathrm{ml}$ of sertaconazole was prepared by dissolving $10 \mathrm{mg}$ of sertaconazole in $5 \mathrm{ml}$ of methanol kept in a $10 \mathrm{ml}$ volumetric flask. Finally, ultrasonication was carried out for $5 \mathrm{~min}$, and volume was made up with methanol. Further, this solution was used to prepare the required dilutions of concentration ranging from $10-60 \mu \mathrm{g} / \mathrm{ml}$ of sertaconazole nitrate.

\section{Analysis of tablets}

Finely ground and powdered tablet equivalent to $10 \mathrm{mg}$ of sertaconazole was ultrsonicated for $20 \mathrm{~min}$ in a $10 \mathrm{ml}$ volumetric flask, having methanol. Finally, volume was made up followed by subsequent filtration using Whatmann filter paper. Drug content in the above solution was determined using the calibration curves of standard sertaconazole.

\section{Method validation}

Specificity: Specificity of the UV spectrophotometric method was evaluated by spectral verification UV spectra for any interference at the absorption maximum of drug because of excipients.

Linearity: Suitable aliquots from $100 \mu \mathrm{g} / \mathrm{ml}$ solution of standard sertaconazole in separate $10 \mathrm{ml}$ volumetric flasks were diluted to obtain concentration of $10-60 \mu \mathrm{g} / \mathrm{ml}$. Dilution up to $10 \mathrm{ml}$ was carried out with methanol. Spectral measurements were done at $260 \mathrm{~nm}, 293 \mathrm{~nm}$ and $302 \mathrm{~nm}$, respectively. Calibration curves were generated by taking the response $v s$. concentration and were later utilized for quantification purpose.

Accuracy and precision: Accuracy of the method using standard addition method was conducted at 80,100 and $120 \%(n=3)$ of the test concentration $(20 \mu \mathrm{g} / \mathrm{ml})$. The amount of drug standard added to the recovery solutions were calculated. The precision (intraday and interday) was assessed by hexaplicate analysis of sertaconazole $(30 \mu \mathrm{g} /$ $\mathrm{ml}$ ) and relative standard deviation (RSD) values were determined. 
Citation: Panda SS, Beg S, Ravi Kumar VVB, Gain A (2017) Spectrophotometric Determination of Sertaconazole Nitrate in Pharmaceutical Dosage Form using Quality by Design (QbD) Framework. J Bioanal Biomed 9: 235-239. doi:10.4172/1948-593X.1000185

\section{Results and Discussion}

The present paper describes a QbD oriented UV spectrophotometric method for analysis of sertaconazole in tablets. Sertaconazole was insoluble in solvents other than methanol. Further, the studies were conducted using methanol as solvent. Standard sertaconazole solution shows absorption maximums $\left(\lambda_{\max }\right)$ at $260 \mathrm{~nm}, 293 \mathrm{~nm}$ and $302 \mathrm{~nm}$, respectively in methanol and were selected as the detection wavelength (Figure 4). CMVs were identified using fish-bone diagram and a C-N-X based risk estimation matrix (Table 1). The various method variables were assigned with total score and selected accordingly for DoE investigation.

Ten randomized experiments as per a CCD were performed on a UV spectrophotometer to investigate the effect of CMVs on the CAA. Details of the experimentation strategy along with obtained results are listed in Table 2.

An experimental investigation was carried out using CCD model utilizing statistical tools and the obtained results were studied through ANOVA, parameter estimates, pareto plot and prediction profiler. The actual versus predicted plot (Figure 5) demonstrated that the obtained experimental results were within the inclusion boundaries, rejecting the null hypothesis. Higher values for $\mathrm{R}^{2}$ indicate the model capability to explain most of the variation. Satisfactory p-values found in ANOVA, and low predicted residual sum of squares (PRESS) values suggested model aptness.

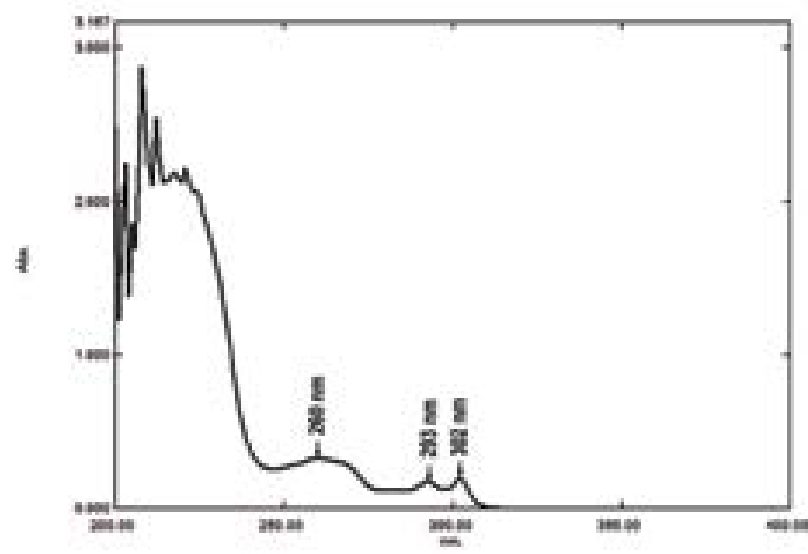

Figure 4: Typical ultra-violet spectra of sertaconazole nitrate.

\begin{tabular}{|l|c|c|c|c|}
\hline Method Variables & Risk Level on CAA & $\begin{array}{c}\text { Final } \\
\text { Score }\end{array}$ & $\mathbf{C , ~ N , \mathbf { X }}$ & Strategy \\
\hline Sampling Interval & 10 & 100 & $\mathrm{X}$ & DoE \\
\hline Scanning Speed & 10 & 100 & $\mathrm{X}$ & DoE \\
\hline Solvent & 5 & 50 & $\mathrm{C}$ & Controlled \\
\hline $\begin{array}{l}\text { Detection } \\
\text { Wavelength }\end{array}$ & 4 & 40 & $\mathrm{C}$ & $269 \mathrm{~nm}$ \\
\hline Sample Integrity & 4 & 40 & $\mathrm{~N}$ & $\begin{array}{c}\text { Quality } \\
\text { Assessed }\end{array}$ \\
\hline $\begin{array}{l}\text { Sample } \\
\text { Preparation }\end{array}$ & 3 & 30 & $\mathrm{C}$ & Controlled \\
\hline $\begin{array}{l}\text { Detector } \\
\text { Equilibration }\end{array}$ & 2 & 20 & $\mathrm{C}$ & Controlled \\
\hline
\end{tabular}

C, N, X-Controlled, Noise, Experimental; Risk Level: 1-Negligible, 5-Low, 10-High; Final Score $=($ Risk Level of $\mathrm{CMV} \times 10)$

Table 1: Risk assessment matrix based on C-N-X approach.
Observed p-value less than 0.05 suggest a non-zero value of slope indicated effective parameter estimate study. The Pareto plot (Figure 6) revealed that both the CMVs had some significant effect on response absorbance.

Investigations through the different statistical parameters justified the suitability of the selected mathematical model. The prediction expressions (Table 3 ) for adopted model are in the following polynomial equation form:

\begin{tabular}{|c|c|c|}
\hline Experiment No. & Sampling Interval (A) & Scanning Speed (B) \\
\hline 1 & 1 & 0 \\
\hline 2 & 0 & -1 \\
\hline 3 & -1 & 0 \\
\hline 4 & 0 & 1 \\
\hline 5 & -1 & 1 \\
\hline 6 & -1 & -1 \\
\hline 7 & 0 & 0 \\
\hline 8 & 1 & -1 \\
\hline 9 & 0 & 0 \\
\hline 10 & 1 & 1 \\
\hline Coded Level & \multicolumn{2}{|c|}{} \\
\hline 1(Low) & $0.5 \mathrm{~nm}$ & Slow \\
\hline 0(Nominal) & $1.0 \mathrm{~nm}$ & Medium \\
\hline+ (High) & $2.0 \mathrm{~nm}$ & Fast \\
\hline
\end{tabular}

Table 2: Experimental design matrix depicting levels of CMVs investigated.

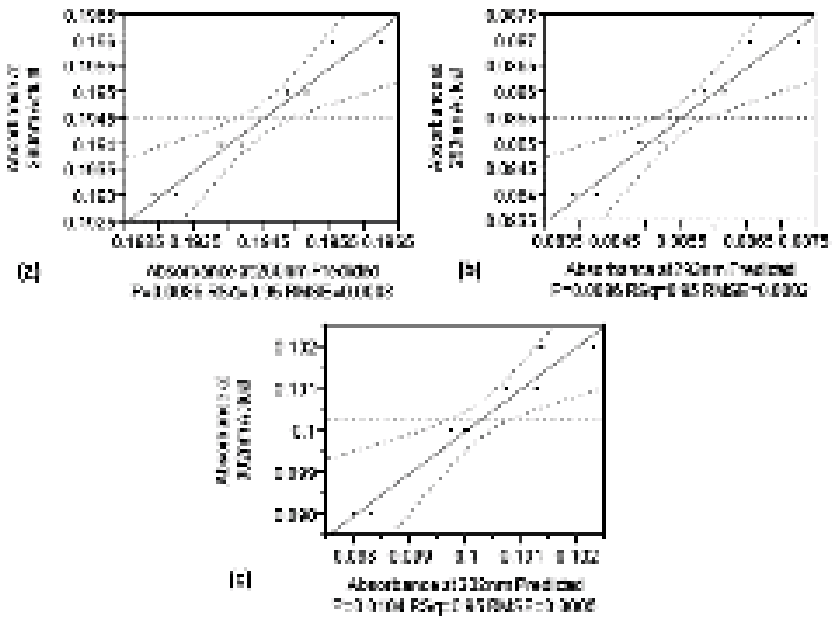

Figure 5: Actual vs. predicted plot for response at (a) $260 \mathrm{~nm}$, (b) $293 \mathrm{~nm}$ and (c) $302 \mathrm{~nm}$

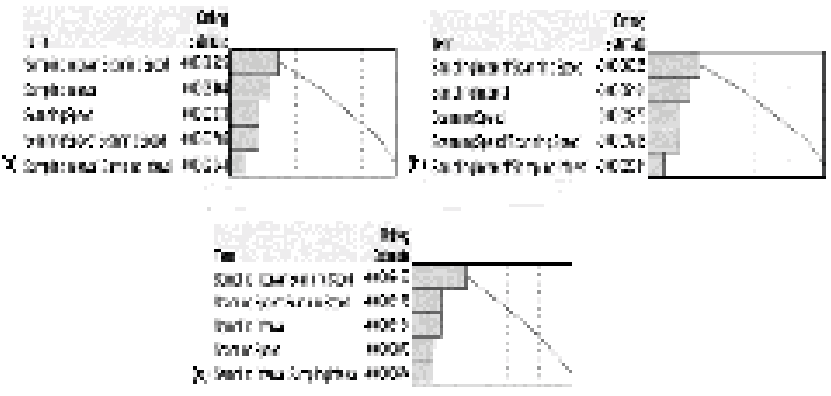

Figure 6: Typical Pareto charts showing effect of CMVs on CAA. 
Citation: Panda SS, Beg S, Ravi Kumar VVB, Gain A (2017) Spectrophotometric Determination of Sertaconazole Nitrate in Pharmaceutical Dosage Form using Quality by Design (QbD) Framework. J Bioanal Biomed 9: 235-239. doi:10.4172/1948-593X.1000185

\section{Absorbance $(Y)=\beta_{0}+\beta_{1} A+\beta_{2} B+\beta_{3} A B+\beta_{4} A^{2}+\beta_{5} B^{2}$}

Where, $A=$ Sampling Interval, $B=$ Scanning Speed, $\beta_{0}=$ coefficient of intercept, $\beta_{1}-\beta_{5}=$ coefficients of model terms

The 3D surface profiler and 2-D contour analysis was carried out for interpretation as well as optimization purpose. The contours obtained (Figure 7A-C) for optimized conditions advocated proceeding to the study with selected center values for both the CMVs. A common pattern was noticed for both the CMVs in all the three 3-D response surfaces for the response Y1, Y2 and Y3 (Figure 8A-D). A curvilinear increase in response was noticed with a gradual increase in scanning speed at low levels of sampling interval. Similarly, a slight increase in

\begin{tabular}{|c|c|c|c|}
\hline \multirow{2}{*}{$\begin{array}{c}\text { Coefficient } \\
\text { Code }\end{array}$} & \multicolumn{3}{|c|}{ Polynomial coefficients } \\
\cline { 2 - 4 } & $\begin{array}{c}\text { Absorbance } \\
\text { at 260nm }\left(\mathbf{Y}_{1}\right)\end{array}$ & $\begin{array}{c}\text { Absorbance } \\
\text { at 293nm }\left(\mathbf{Y}_{2}\right)\end{array}$ & $\begin{array}{c}\text { Absorbance } \\
\text { at 302nm }\left(\mathbf{Y}_{3}\right)\end{array}$ \\
\hline$\beta_{0}$ & 0.1951429 & 0.0861429 & 0.1012857 \\
\hline$\beta_{1}$ & -0.000667 & -0.000667 & -0.000667 \\
\hline$\beta_{2}$ & 0.0005 & 0.0005 & 0.0005 \\
\hline$\beta_{3}$ & -0.001 & -0.001 & -0.0015 \\
\hline$\beta_{4}$ & -0.000286 & -0.000286 & -0.000571 \\
\hline$\beta_{5}$ & -0.000786 & -0.000786 & -0.001071 \\
\hline
\end{tabular}

Table 3: Coefficients of prediction expressions obtained for the selected experimental domain.

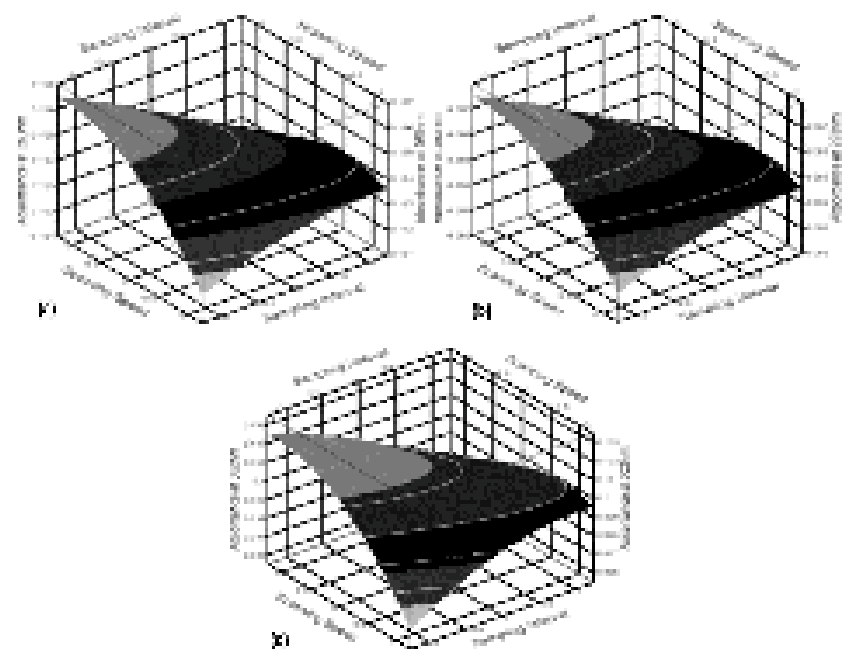

Figure 7: 3-D surface profilers obtained for response at (a) $260 \mathrm{~nm}$, (b) 293 $\mathrm{nm}$ and (c)

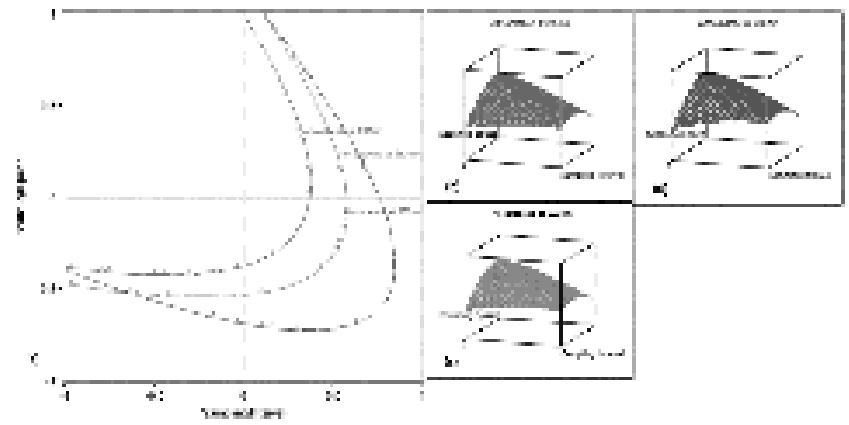

Figure 8: Design space obtained for optimized experimental conditionst.

\begin{tabular}{|c|c|c|c|}
\hline \multirow{2}{*}{\begin{tabular}{|l} 
Parameters \\
Wavelength $(\mathrm{nm})$
\end{tabular}} & \multicolumn{3}{|l|}{ Observed Values } \\
\hline & 260 & 293 & 302 \\
\hline Linearity range $(\mu \mathrm{g} / \mathrm{ml})$ & $10-60$ & $10-60$ & $10-60$ \\
\hline $\begin{array}{l}\text { Sandell's sensitivity }(\mu \mathrm{g} / \\
\left.\mathrm{cm}^{2} / 0.001 \mathrm{AU}\right)\end{array}$ & 0.117 & $\ldots$ & $\cdots$ \\
\hline $\begin{array}{l}\text { Molar extinction } \\
\text { coefficient }\left(\mathrm{L} \mathrm{mol}^{-1} \mathrm{~cm}^{-1}\right)\end{array}$ & $4.256 \times 10^{3}$ & $\cdots$ & $\cdots$ \\
\hline Regression equation & $Y=0.010 x-0.0152$ & $Y=0.006 x-0.042$ & $Y=0.007 x-0.038$ \\
\hline Corelation coefficient $\left(\mathrm{R}^{2}\right)$ & 0.999 & 0.999 & 0.999 \\
\hline $\begin{array}{l}\text { Precision (\% R.S.D.) } \\
\text { Intra-day } \\
\text { Inter-day }\end{array}$ & $\begin{array}{l}0.516 \\
0.938 \\
\end{array}$ & $\begin{array}{l}0.509 \\
0.577 \\
\end{array}$ & $\begin{array}{l}0.632 \\
0.345 \\
\end{array}$ \\
\hline $\begin{array}{l}\text { Accuracy (\% Recovery } \\
{ }^{\dagger} \pm \text { S.D.) } \\
80 \% \\
100 \% \\
120 \% \\
\end{array}$ & $\begin{array}{l}99.7 \pm 0.207 \\
99.37 \pm 0.607 \\
99.0 \pm 0.839\end{array}$ & $\begin{array}{l}99.32 \pm 0.427 \\
99.4 \pm 0.372 \\
98.27 \pm 0.388\end{array}$ & $\begin{array}{l}98.27 \pm 0.722 \\
99.57 \pm 0.231 \\
98.14 \pm 0.792\end{array}$ \\
\hline $\begin{array}{l}\text { \% Range of error } \\
0.05 \text { confidence limits } \\
0.01 \text { confidence limits }\end{array}$ & $\begin{array}{l} \pm 0.12 \\
\pm 0.158\end{array}$ & $\begin{array}{l} \pm 0.064 \\
\pm 0.084\end{array}$ & $\begin{array}{l} \pm 0.088 \\
\pm 0.116\end{array}$ \\
\hline
\end{tabular}

R.S.D.-Relative Standard Deviation; S.D.-Standard Deviation; A.U.-Absorbance Units, ${ }^{\dagger}$ is average of triplicate determinations at each level

Table 4: Summary of observed results for the developed analytical method.

response was noticed at low levels of scanning speed and increasing levels of sampling interval. However, a minimum response was noticed at low levels of both the CMVs.

The optical features of the developed analytical method were found satisfactory (Table 4). Specificity as well as selectivity of the method was achieved due to non-interference of the commonly used excipients present in the tablets. Linearity over concentration range of $10-60 \mu \mathrm{g} / \mathrm{ml}$ of sertaconazole was established. The recovery of standard sertaconazole from tablets was found to be within $98.63-99.68 \%(n=3)$ at all the $\lambda_{\max }$. The results of accuracy $(98.14-99.7 \%)$ and precision (\%RSD $<2$ ) were acceptable. The results from the validation study indicate the method is interference free and complies with the ICH requirements.

\section{Conclusion}

A robust UV spectrophotometric method was developed for determination of sertaconazole in tablets, using the systematic QbD approach. Two CMVs viz. sampling interval and scanning speed required systematic investigation and optimization to obtain a quality analytical method. The results of the study suggested method novelty, simplicity, accuracy and preciseness. The obtained values of method validation as per statistical studies indicated fitness of the method for routine analysis purpose. Lack of interference in analysis due to commonly used excipients established method specificity. Therefore, this QbD based analytical method can be employed for determination of sertaconazole in pharmaceuticals.

\section{Acknowledgments}

The authors are thankful to Glenmark Pharmaceuticals Ltd., India for providing the gift sample of sertaconazole nitrate standard drug and Roland Institute of Pharmaceutical Sciences, Berhampur-10, Odisha, India for providing the research facilities.

\section{References}

1. Carrillo-Munoz AJ, Tur-Tur C, Giusiano G, Marcos-Arias C, Eraso E, et al (2013) Sertaconazole: an antifungal agent for the topical treatment of superficial candidiasis. Expert Rev Anti Infect Ther 11: 347-358.

2. Goldust M, Rezaee E, Raghifar R (2013) Treatment of seborrheic dermatitis, comparison of sertaconazole $2 \%$ cream versus ketoconazole $2 \%$ cream. Ir J Med Sci 182: 703-706. 
Citation: Panda SS, Beg S, Ravi Kumar VVB, Gain A (2017) Spectrophotometric Determination of Sertaconazole Nitrate in Pharmaceutical Dosage Form using Quality by Design (QbD) Framework. J Bioanal Biomed 9: 235-239. doi:10.4172/1948-593X.1000185

3. Siras SS, Mandlik SK (2016) UV-Spectrophotometric Method Development and Validation of Sertaconazole Nitrate in Bulk and Tablet Dosage Form. Inventi Rapid: Pharm Anal Qual Assur 3: 1-5.

4. Gouda AA, El-Sheikh R, Amin AS, Ibrahim SH (2016) Optimized and validated spectrophotometric determination of two antifungal drugs in pharmaceutical formulations using an ion-pair complexation reaction. J Talibah Univ Sci 10 26-37.
5. Muzaffar F Singh UK (2016) RP-HPLC and UV spectrophotometric methods for estimation of sertaconazole nitrate in microemulsion. J Chem Pharm Res 8: 740-745.

6. Godfrey AB, Kenett RS (2007) Joseph M. Juran, a perspective on past contributions and future impact. Qual Reliab Engg Int 23: 653-663.

7. International Conference on Harmonization (ICH) (2005) Validation of Analytical Procedures: Text and Methodology. IFPMA, Geneva. 